\title{
Impaired fasting glucose as an independent risk factor for hypertension among healthy middle-aged Japanese subjects with optimal blood pressure: the Yuport Medical Checkup Centre retrospective cohort study
}

\author{
Masaaki Morio $^{1 *}$, Machiko Inoue $^{2}$, Kazuo Inoue $^{3}$ and Kimihiko Akimoto ${ }^{4}$
}

\begin{abstract}
Background: This study aimed at investigating whether impaired fasting glucose (IFG) is an independent risk factor for incident hypertension among middle-aged Japanese subjects with optimal blood pressure (OBP).

Findings: This retrospective cohort study was conducted in 2943 non-diabetic and non-hypertensive subjects aged 40-64 years, who participated in a voluntary health check-up program during the baseline (1998-2002) and follow-up periods (2002-2006). A multiple logistic regression model was utilized to calculate the odds ratio (OR) of incident hypertension among men and women with IFG and OBP. OBP was defined as systolic blood pressure (SBP) $<120 \mathrm{mmHg}$ and diastolic blood pressure (DBP) $<80 \mathrm{mmHg}$, with no known history of hypertension. In this study, hypertension was defined as SBP $\geq 140 \mathrm{mmHg}$ and DBP $\geq 90 \mathrm{mmHg}$ or by a self-reported clinical diagnosis of hypertension. After the mean follow-up period of 5.6 years, the incidence of hypertension in men and women was 5.7\% (73/1270) and 3.8\% (62/1673), respectively. The age-adjusted ORs for incident hypertension in men and women with IFG were 1.95 (95\% Cl, 1.21-3.15) and 3.54 (95\% Cl, 2.00-6.27), respectively. After adjusting for age, systolic blood pressure, body mass index, total cholesterol, triglyceride, high-density lipoprotein cholesterol, and uric acid, the ORs for hypertension were 1.66 (95\% Cl; 1.02-2.70) for men and 2.62 (95\% Cl, 1.45-4.73) for women.

Conclusion: The study results show that IFG may act as an independent risk factor for developing hypertension in individuals with OBP.
\end{abstract}

Keywords: Fasting plasma glucose, Impaired fasting glucose, Optimal blood pressure, Hypertension

\section{Background}

Hypertension and diabetes are the leading morbidities affecting the general population, often concurrently, as there is substantial overlap between hypertension and diabetes in both etiology and disease mechanism [1-3]. The association between elevated blood pressure and dysglycemia has also recently been of interest [4].

In 1997, the American Diabetes Association (ADA) introduced the term 'impaired fasting glucose' (IFG),

\footnotetext{
* Correspondence: masaaki_morio@kochi-u.ac.jp

'Department of Family Medicine, Kochi Medical School, Kohasu, Oko-cho, Nankoku, Kochi, Japan

Full list of author information is available at the end of the article
}

defined as a fasting plasma glucose (FPG) level of 110$125 \mathrm{mg} / \mathrm{dL}\left(\mathrm{IFG}^{1997}\right.$ ), to describe a prediabetic state of dysglycemia [5]. Accordingly, researchers began to examine whether IFG may be an independent risk factor for the development of hypertension as well. However, previous findings were inconsistent [6-10]. Although IFG and elevated FPG emerged as independent risk factors for the development of hypertension in 3 prospective studies [6-8], this relationship was not found in 2 other studies after adjusting for body mass index (BMI) or waist circumference $[9,10]$.

Central obesity is a well-known risk factor for both diabetes and hypertension [3,11], and is thought to be a 
common pathway whereby obesity affects both diseases $[1,2]$. Simone et al. reported that abdominal obesity was a major predictor of the development of hypertension even at optimal blood pressure (OBP) [8], which suggests that atherogenic risk factors could play a major role in atherosclerosis even when blood pressure is low or at optimal levels.

The association of IFG and hypertension in those with OBP has not been investigated thoroughly. Furthermore, although the ADA lowered the FPG concentration range for diagnosis of IFG from $110-125 \mathrm{mg} / \mathrm{dL}$ to $100-125$ $\mathrm{mg} / \mathrm{dL}$ in 2003 [9], there have been no studies conducted to determine whether this new criterion for IFG is predictive of the development of hypertension.

In this study, we investigated whether the new criterion for IFG could be considered as an independent risk factor for incident hypertension among healthy middleaged Japanese subjects with OBP.

\section{Methods}

\section{Study subjects}

We used a health screening program cohort of 13,734 healthy middle-aged Japanese subjects, who received routine medical check-ups during the baseline period between April 1998 and March 2002 at the Yuport Medical Checkup Centre in Tokyo, which has been described previously [12-15]. If subjects received more than 1 check-up during the baseline period, the initial check-up data were used. The 4-year follow-up period ended between April 2002 and March 2006, and the 6,290 subjects who did not have follow-up data were excluded. If subjects received more than 1 check-up during the follow-up period, all data were used to identify incident hypertension. This study excluded 424 subjects who had a baseline FPG $\geq 126 \mathrm{mg} / \mathrm{dL}, \mathrm{HbA1c} \geq 6.5 \%$, or self-reported clinically diagnosed diabetes, as well as 4067 subjects with a baseline SBP $\geq 120 \mathrm{mmHg}$, DBP $\geq 80$ $\mathrm{mmHg}$, or self-reported clinical diagnosis of hypertension. The remaining 2943 non-diabetic and non-hypertensive patients were included as study subjects (mean follow-up, $5.6 \pm 1.6$ years).

\section{Clinical and laboratory procedures}

All medical check-ups followed the same procedures during both the baseline and follow-up periods. Resting blood pressure was measured twice by well-trained nurses using a mercury column sphygmomanometer, after each subject sat quietly for at least $5 \mathrm{~min}$. The average of the 2 measurements was used for analysis.

All blood samples were collected after an overnight fast. Blood samples were measured on the same autoanalyzer in the laboratory at the medical center. Plasma glucose levels were measured using the hexokinaseG6PD method (Denka Seiken, Niigata, Japan) with an interassay coefficient of variation $(\mathrm{CV}) \leq 3.0 \%$. Hemoglobin A1c (HbA1c) levels were measured using a latex immunoagglutination assay method (Determiner HbA1c, Kyowa Medex, Tokyo, Japan) with an interassay CV of $1.7-2.1 \%$, which was aligned with the units used by the Japan Diabetes Society (JDS). The JDS value for HbA1c was converted into National Glycohemoglobin Standardization Program (NGSP) units in this study, which was calculated by the formula: HbA1c (NGSP) = HbA1c (JDS) + 0.4 [14]. Other blood tests, total cholesterol (TC), highdensity lipoprotein cholesterol (HDL-C), triglycerides (TG), creatinine $(\mathrm{Cr})$, and uric acid (UA), were measured using enzymatic methods (reagents supplied by Daiichi Pure Chemicals, Tokyo, Japan). Low-density lipoprotein cholesterol (LDL-C) was calculated by the Friedewald equation [16].

In accordance with the Private Information Protection Law, information that might identify subjects was kept confidential. This study was approved by the review board of the Yuport Medical Checkup Centre, and informed consent for anonymous participation in this study was obtained from each subject at every check-up.

\section{Diagnosis of hypertension and IFG}

The Seventh Report of the Joint National Committee on Prevention, Detection, Evaluation, and Treatment of High Blood Pressure (JNC 7) criteria were utilized [17] to define OBP as SBP $<120 \mathrm{mmHg}$ and DBP $<80 \mathrm{mmHg}$. Hypertension was defined as SBP $\geq 140 \mathrm{mmHg}$ and DBP $\geq 90 \mathrm{mmHg}$, or as self-reported clinically diagnosed hypertension. FPG categories were defined by using the 2003 ADA criteria [18] as normal fasting glucose (NFG); FPG $\leq 99 \mathrm{mg} / \mathrm{dL}$ and IFG; FPG of $100-125 \mathrm{mg} / \mathrm{dL}$.

\section{Statistical analysis}

Baseline characteristics for all subjects are presented as mean \pm standard deviation (SD). A Students t-test or chi-square test was used to test for differences in mean values and frequencies between men and women. We calculated the odds ratios (ORs) and 95\% confidence intervals (CIs) to assess the association between IFG (versus NFG) at baseline and the incidence of hypertension at follow-up using multiple logistic regression analyses. We used two multivariable models: model 1, adjusted for age; and model 2, adjusted for age, BMI, SBP, TC, TG, HDL-C and UA. All reported $P$-values were two-tailed, and $P<0.05$ was considered statistically significant. All statistical analyses were performed using SPSS version 19 (SPSS Inc., Japan).

\section{Results}

The baseline characteristics of subjects and the development of hypertension by sex are given in Table 1 . Women were older and had significantly lower SBP, DBP, TG, Cr, 
Table 1 Baseline characteristics and incidence of hypertension

\begin{tabular}{lllll}
\hline & Total & Men & Women & \\
& $\mathbf{N = 2 9 4 3}$ & $\begin{array}{l}\mathbf{N = 1 2 7 0} \\
\mathbf{( 4 3 . 2 \% )}\end{array}$ & $\begin{array}{l}\mathbf{N = 1 6 7 3} \\
\mathbf{( 5 6 . 8 \% )}\end{array}$ & P-value $^{*}$ \\
\hline Age (years) & $51.7 \pm 6.7$ & $51.0 \pm 6.7$ & $52.2 \pm 6.7$ & $<0.001$ \\
BMI (kg/m²) & $22.1 \pm 2.6$ & $22.7 \pm 2.6$ & $21.5 \pm 2.6$ & $<0.001$ \\
SBP (mmHg) & $107.5 \pm 7.9$ & $108.8 \pm 7.2$ & $106.5 \pm 8.3$ & $<0.001$ \\
DBP (mmHg) & $65.8 \pm 6.2$ & $67.5 \pm 5.6$ & $64.5 \pm 6.4$ & $<0.001$ \\
FPG (mg/dL) & $92.8 \pm 8.5$ & $96.1 \pm 8.5$ & $90.4 \pm 7.5$ & $<0.001$ \\
HbA1c (\%) & $5.4 \pm 0.4$ & $5.4 \pm 0.4$ & $5.4 \pm 0.4$ & 0.517 \\
TC (mg/dL) & $201.6 \pm 33.7$ & $194.8 \pm 32.5$ & $206.7 \pm 33.7$ & $<0.001$ \\
TG (mg/dL) & $102.6 \pm 62.0$ & $124.8 \pm 76.3$ & $85.7 \pm 40.9$ & $<0.001$ \\
HDL-C (mg/dL) & $60.3 \pm 15.6$ & $52.7 \pm 13.3$ & $66.0 \pm 14.7$ & $<0.001$ \\
LDL-C (mg/dL) & $120.8 \pm 30.1$ & $117.1 \pm 30.2$ & $123.6 \pm 30.8$ & $<0.001$ \\
Cr (mg/dL) & $0.70 \pm 0.15$ & $0.82 \pm 0.13$ & $0.61 \pm 0.09$ & $<0.001$ \\
UA (mg/dL) & $5.0 \pm 1.3$ & $5.9 \pm 1.2$ & $4.4 \pm 0.9$ & $<0.001$ \\
Follow-up (years) & $5.6 \pm 1.6$ & $5.7 \pm 1.5$ & $5.5 \pm 1.6$ & $<0.001$ \\
HT (\%) & $135(4.6 \%)$ & $73(5.7 \%)$ & $62(3.7 \%)$ & 0.010 \\
\hline
\end{tabular}

Data are presented as mean \pm SD or number (\%).

"Mean or frequency (\%) difference between men and women.

$\mathrm{BMI}$, body mass index; SBP, systolic blood pressure; DBP, diastolic blood pressure; FPG, fasting plasma glucose; $\mathrm{HbA1c}$, glycosylated hemoglobin; TC, total cholesterol; TG, triglyceride; $\mathrm{HDL}-\mathrm{C}$, high density lipoprotein cholesterol; LDL-C, low density lipoprotein cholesterol; $\mathrm{Cr}$, creatinine; UA, uric acid; $\mathrm{HT}$, hypertension.

and UA, while they had higher TC, HDL-C, and LDL-C than men. The HbA1c level was not statistically different between the sexes. The incidence of hypertension at the mean follow-up time of 5.6 years was $5.7 \%$ and $3.7 \%$ in men and women, respectively.
The incidence of hypertension in the FPG categories and the multivariate-adjusted ORs for developing hypertension in subjects with OBP and IFG when compared with NFG by sex are given in Table 2. The age-adjusted OR (shown as Model 1) for the development of hypertension was 1.95 (95\% CI, 1.21-3.15) and 3.54 (95\% CI, 2.00-6.27) for men and women, respectively. After adjusting for age, BMI, SBP, TC, TG, HDL-C and UA, the OR (shown as Model 2) for hypertension was 1.66 (95\% CI, 1.02-2.70) and 2.62 (95\% CI, 1.45-4.73) for men and women, respectively. Age and SBP in both sexes and BMI in women also remained as predictors of incident hypertension. The IFG in both sexes was significantly and positively associated with the risk of hypertension.

\section{Discussion}

The main finding of this study was that IFG may act as an independent risk factor for the development of hypertension in middle-aged healthy Japanese subjects with OBP. In 1997, after the ADA introduced IFG ${ }^{1997}$ [5], various prospective studies have demonstrated that impaired glucose tolerance (IGT), another form of dysglycemia, was an independent risk factor for incident hypertension [4,19-21]. However, the results from previous investigations of the association between IFG $^{1997}$ or elevated FPG and the risk for hypertension are conflicting [6-10], with 3 positive and 2 negative results.

This study has some methodological strengths as compared with previous studies. First, our cohort population was relatively large and had a single homogenous ethnicity. Second, all subjects were examined at one site, and

Table 2 Odds ratio for incident hypertension in middle-aged Japanese subjects with OBP by sex

\begin{tabular}{|c|c|c|c|c|c|}
\hline & \multirow[t]{2}{*}{ FPG categories at baseline } & \multicolumn{2}{|c|}{ Men } & \multicolumn{2}{|c|}{ Women } \\
\hline & & \multicolumn{2}{|c|}{ Incidence of hypertension, (\%) } & \multicolumn{2}{|c|}{ Incidence of hypertension, (\%) } \\
\hline & NFG & \multicolumn{2}{|c|}{$40 / 848(4.5 \%)$} & \multicolumn{2}{|c|}{$43 / 1453(2.9 \%)$} \\
\hline & \multirow[t]{2}{*}{ IFG } & \multicolumn{2}{|c|}{$33 / 349(8.6 \%)$} & \multicolumn{2}{|c|}{$19 / 158(10.7 \%)$} \\
\hline & & OR $(95 \% \mathrm{Cl})$ & $P$-value & OR $(95 \% \mathrm{Cl})$ & $P$-value \\
\hline Model $1^{*}$ & IFG vs. NFG & $1.95(1.21-3.15)$ & 0.006 & $3.54(2.00-6.27)$ & $<0.001$ \\
\hline \multirow[t]{8}{*}{ Model $2^{* *}$} & IFG vs. NFG & $1.66(1.02-2.70)$ & 0.043 & $2.62(1.45-4.73)$ & 0.001 \\
\hline & Age (years) & $1.04(1.00-1.08)$ & 0.046 & $1.05(1.00-1.10)$ & 0.042 \\
\hline & $\mathrm{BMI}\left(\mathrm{kg} / \mathrm{m}^{2}\right)$ & $1.06(0.95-1.17)$ & 0.332 & $1.17(1.06-1.29)$ & 0.002 \\
\hline & $\mathrm{SBP}(\mathrm{mmHg})$ & $1.12(1.07-1.18)$ & $<0.001$ & $1.09(1.05-1.14)$ & $<0.001$ \\
\hline & TC (per 10 mg/dL) & $1.03(0.95-1.11)$ & 0.478 & $0.94(0.86-1.04)$ & 0.228 \\
\hline & TG (per 10 mg/dL) & $1.03(1.00-1.06)$ & 0.079 & $1.06(0.99-1.12)$ & 0.079 \\
\hline & $\mathrm{HDL}-\mathrm{C}$ (per 10 mg/dL) & $1.00(0.80-1.25)$ & 1.000 & $0.96(0.75-1.22)$ & 0.727 \\
\hline & $\mathrm{UA}(\mathrm{mg} / \mathrm{dL})$ & $1.10(0.90-1.34)$ & 0.361 & $1.17(0.89-1.55)$ & 0.268 \\
\hline
\end{tabular}

${ }^{*}$ Model 1: adjusted for age.

${ }^{* *}$ Model 2: adjusted for age, BMI, SBP, TC, TG, HDL-C, and UA.

FPG, fasting plasma glucose; NFG, normal fasting glucose; IFG, impaired fasting glucose. 
laboratory samples were measured using the same method both at baseline and follow-up.

Our study had several limitations. First, we could not include several possible confounding variables that are known hypertension risk factors, such as plasma insulin, family history, alcohol and salt consumption, smoking, waist circumference, and socioeconomic status. These variables could be relevant for explaining the relationship between IFG and the risk for hypertension. Second, incident hypertension was defined by a single basal measurement of blood pressure, which was repeated at follow-up, or by a self-reported clinical diagnosis of hypertension. Third, the study subjects were men and women who had regular check-ups at a medical center in Tokyo, and they may be a healthier subset of the general population.

In summary, this study demonstrated an association between IFG and the development of hypertension among relatively lean Japanese subjects with OBP after the ADA lowered the FPG threshold value for IFG from 110 to $100 \mathrm{mg} / \mathrm{dL}$ in 2003. The findings may help clinicians identify those who are at higher risk for hypertension.

\section{Competing interests}

The authors declare that they have no competing interests.

\section{Authors' contributions}

$\mathrm{MM}, \mathrm{MI}, \mathrm{Kl}$, and $\mathrm{KA}$ conceived and designed the experiments. KA was responsible for data collection and performed the experiments. MM performed the statistical analysis and drafted the manuscript. MI and Kl provided the critical revision. All authors have given final approval of the version to be published.

\section{Acknowledgements}

We are grateful to Mrs. Tetsuya Hayashi and Kiyonori Uchiyama for their assistance with data collection.

\section{Author details}

'Department of Family Medicine, Kochi Medical School, Kohasu, Oko-cho, Nankoku, Kochi, Japan. ${ }^{2}$ Department of Community Medicine, Teikyo University School of Medicine, 2-11-1 Kaga, Itabashi-ku, Tokyo, Japan. ${ }^{3}$ Department of Community Medicine, Chiba Medical Center, Teikyo University School of Medicine, 3426-3, Anesaki, Ichihara, Chiba, Japan. ${ }^{4}$ Akimoto Occupational Health Consultant Office, 4-7-22 Kudan-minami, Chiyoda-ku, Tokyo, Japan.

Received: 11 June 2013 Accepted: 16 December 2013 Published: 20 December 2013

\section{References}

1. Cheung BM, Li C: Diabetes and hypertension: is there a common metabolic pathway? Curr Atheroscler Rep 2012, 14(2):160-166.

2. Cheung BM: The hypertension-diabetes continuum. $J$ Cardiovasc Pharmacol 2010, 55(4):333-339.

3. Mokdad AH, Ford ES, Bowman BA, Dietz WH, Vinicor F, Bales VS, Marks JS: Prevalence of obesity, diabetes, and obesity-related health risk factors, 2001. JAMA 2003, 289(1):76-79.

4. Cheung BM, Wat NMS, Tso AWK, Tam S, Thomas GN, Leung GM, Tse HF, Woo J, Janus ED, Lau CP, Lam TH, Lam KS: Association between raised blood pressure and dysglycemia in Hong Kong Chinese. Diabetes Care 2008, 31(9):1889-1891.
5. The Expert Committee on the Diagnosis and Classification of Diabetes Mellitus: Report of the expert committee on the diagnosis and classification of diabetes mellitus. Diabetes Care 1997, 20(7):1183-1197.

6. Bjørnholt JV, Erikssen G, Kjeldsen SE, Bodegard J, Thaulow E, Erikssen J: Fasting blood glucose is independently associated with resting and exercise blood pressures and development of elevated blood pressure. J Hypertens 2003, 21(7):1383-1389.

7. Fagot-Campagna A, Balkau B, Simon D, Ducimetiere P, Eschwege E: Is insulin an independent risk factor for hypertension? The Paris Prospective Study. Int J Epidemiol 1997, 26(3):542-550.

8. Suematsu C, Hayashi T, Fujii S, Endo G, Tsumura K, Okada K, Morii H: Impaired fasting glucose and the risk of hypertension in Japanese men between the 1980s and the 1990s. The Osaka Health Survey. Diabetes Care 1999, 22(2):228-232.

9. Wang W, Lee ET, Fabsitz RR, Devereux R, Best L, Welty TK, Howard BV: A longitudinal study of hypertension risk factors and their relation to cardiovascular disease: the strong heart study. Hypertension 2006, 47(3):403-409.

10. Levin G, Kestenbaum B, Ida Chen YD, Jacobs DR Jr, Psaty BM, Rotter Jl, Siscovick DS, de Boer IH: Glucose, insulin, and incident hypertension in the multi-ethnic study of atherosclerosis. Am J Epidemiol 2010, 172(10):1144-1154

11. Davy KP, Hall JE: Obesity and hypertension: two epidemics or one? Am J Physiol Regul Intergr Comp Physiol 2004, 286(5):R803-R813.

12. Inoue K, Kashima S, Ohara C, Matsumoto M, Akimoto K: Concordance of two diabetes diagnostic criteria using fasting plasma glucose and hemoglobin A1c: the Yuport Medical Checkup Centre study. PLoS One 2012, 7(10):e47747.

13. Inoue $M$, Inoue $K$, Akimoto $K$ : Effects of age and sex in the diagnosis of type 2 diabetes using glycated haemoglobin in Japan: the Yuport Medical Checkup Centre study. PLoS One 2012, 7(7):e40375.

14. Inoue $K$, Matsumoto $M$, Akimoto K: Fasting plasma glucose and HbA1C as risk factors for type 2 diabetes. Diabet Med 2008, 25(10):1157-1163.

15. Inoue K, Matsumoto M, Akimoto K: The threshold for definition of impaired fasting glucose in a Japanese population. Diabet Med 2009, 26(11):1175-1178.

16. Friedewald WT, Levy RI, Fredrickson DS: Estimation of the concentration of low-density lipoprotein cholesterol in plasma, without use of the preparative ultracentrifuge. Clin Chem 1972, 18:499-502.

17. Chobanian AV, Bakris GL, Black HR, Cushman WC, Green LA, Izzo JL Jr, Jones DW, Materson BJ, Oparil S, Wright JT Jr, Roccella EJ, National High Blood Pressure Education Program Coordinating Committee: The seventh report of the joint national committee on prevention, detection, evaluation, and treatment of high blood pressure: the JNC 7 report. JAMA 2003, 289(19):2560-2572.

18. Genuth S, Alberti KG, Bennett P, Buse J, Defronzo R, Kahn R: Follow-up report on the diagnosis of diabetes mellitus. Diabetes Care 2003, 26(11):3160-3167.

19. Modan M, Halkin H, Almog S, Lusky A, Eshkol A, Shefi M, Shitrit A, Fuchs Z: Hyperinsulinemia. A link between hypertension obesity and glucose intolerance. J Clin Invest 1985, 75(3):809-817.

20. de Simone G, Devereux RB, Chinali M, Roman MJ, Best LG, Welty TK, Lee ET, Howard BV: Risk factors for arterial hypertension in adults with initial optimal blood pressure: the strong heart study. Hypertens 2006, 47(2):162-167.

21. Boyko EJ, Barr EL, Zimmet PZ, Shaw JE: Two-hour glucose predicts the development of hypertension over 5 years: the AusDiab study. J Hum Hypertens 2008, 22(3):168-176.

doi:10.1186/1758-5996-5-81

Cite this article as: Morio et al:: Impaired fasting glucose as an independent risk factor for hypertension among healthy middle-aged Japanese subjects with optimal blood pressure: the Yuport Medical Checkup Centre retrospective cohort study. Diabetology \& Metabolic Syndrome 2013 5:81. 